El bien universal y la buena conciencia: análisis de los conceptos de la conciencia del deber puro y la conciencia moral actuante en la Fenomenología del espíritu y la Filosofía del derecho de Hegel

\title{
MAVERICK Díaz
}

Pontificia Universidad Católica del Perú

Resumen: El presente trabajo tiene como objetivo hacer un análisis de la dinámica entre los conceptos en juego en el tránsito de la moralidad a la eticidad en la filosofía del espíritu objetivo de Hegel. El recurso hermenéutico y conceptual al que apelamos es la complementación entre las lecturas de los movimientos dialécticos de la "Moralidad" en la Filosofía del derecho y de la "Moralidad" en el capítulo sobre el espíritu en la Fenomenología del espíritu. En primer lugar, desarrollaremos los conceptos de conciencia moral del deber (el bien abstracto) y la buena conciencia (das Gewissen) en la Filosofía del derecho. En segundo lugar, abordaremos, como complemento conceptual, el sentido de tales conceptos (la visión moral del mundo y la buena conciencia o conciencia moral actuante) en la Fenomenología del espíritu. Finalmente, ofreceremos, a partir de la articulación dialéctica de tales conceptos, la formulación y el análisis de lo que significa el tránsito de la moralidad a la eticidad y el surgimiento de la libertad ética como superación de la libertad moral.

Palabras claves: conciencia moral, buena conciencia, eticidad, Hegel, moralidad

Abstract: The present work aims to analyse the dynamics between the concepts at stake within the transition from morality to ethical life in Hegel's "Philosophy of Objective Spirit". Our hermeneutical and conceptual resource consists in the complementary readings of the dialectical movements of "morality" in Hegel's Philosophy of Right and in his Phenomenology of Spirit. First of all, we will examine the concepts of abstract good and conscience (das Gewissen) that appear in the Philosophy of Right. Secondly, we will examine the meaning of such concepts (the moral worldview and conscience or acting moral consciousness) in the Phenomenology of Spirit as complementary concepts. Finally, we offer a formulation and analysis of the meaning of the overall 
transition from morality to ethical life, starting from the dialectic articulation of both concepts, and the emergence of ethical freedom as an overcoming of moral freedom.

Key words: moral worldview, conscience, ethical life, Hegel, morality.

\section{§1. Presentación}

El capítulo de la "Moralidad", tanto en la Filosofía del derecho como en La Fenomenología del espíritu de Hegel, convoca dos conceptos fundamentales en su tratamiento: la conciencia moral del deber o visión moral del mundo, encarnada en la filosofía práctica kantiana, y la conciencia moral actuante o buena conciencia (das Gewissen), figura expresada en la filosofía y literatura post-kantiana, vinculada al idealismo de Fichte y al romanticismo. Si bien los dos conceptos, en ambos textos, se encuentran en secciones denominadas "Moralidad", no solo difieren debido a la ubicación de tal sección en el ordenamiento lógico de Hegel y a la extensión de su tratamiento en ella', sino también debido a su contenido conceptual en específico, aunque no de un modo esencial. En ese sentido, el desarrollo de Hegel sobre la buena conciencia en la Fenomenología puede ayudar a descifrar, con más claridad analítica, qué quiere decir que, en la Filosofía del derecho, la libertad moral, debido a su contradictoria parcialidad, es superada en un punto de vista más concreto y completo de la libertad humana, el cual, asimismo, tiene que ser distinguido y explicado de acuerdo a este trasfondo.

Nuestro trabajo se divide en tres apartados. En primer lugar, abordaremos brevemente el desarrollo de las dos figuras en la Filosofía del derecho (2). Esta lectura, en segundo lugar, será complementada por la reconstrucción de algunos puntos decisivos del desarrollo de ambos conceptos en la sección correspondiente en la Fenomenología del espíritu (3). Finalmente, abordaremos, con los recursos conceptuales obtenidos, el tránsito de la moralidad a la

\footnotetext{
I En la Fenomenología, tal sección corresponde al último momento del capítulo del "Espíritu”: el momento de la identidad dialéctica; en la Filosofía del derecho, corresponde, más bien, al segundo momento del espíritu objetivo: la particularidad. Asimismo, en la Filosofía del derecho, la dinámica de ambos conceptos ocupa solo el tercer apartado de la “Moralidad”, mientras que, en la Fenomenología, ocupa todo el contenido del capítulo.
} 
eticidad y la lógica propia de tal superación para, sobre esta base, presentar una última respuesta acerca de la naturaleza de la eticidad o libertad ética (4).

\section{§ 2. El bien abstracto y la buena conciencia en el capítulo de la "Moralidad" de la Filosofía del derecho}

En el primer parágrafo del tercer momento del punto de vista de la moralidad, "El bien y la conciencia moral", Hegel realiza una afirmación fundamental para situar el desarrollo tanto anterior como posterior de su argumentación. De esta manera, el bien (das Gute) es definido como "unidad del concepto de la voluntad y de la voluntad particular"2. Por un lado, esta aseveración comporta la intención de unificar dialécticamente lo desarrollado hasta aquí acerca de los momentos del concepto de voluntad libre. Por otro lado, tal afirmación marca la pauta para interpretar los momentos que aún están por venir. Surgirá, en primer lugar, una manera de comprender la unidad de tal bien que, en vista aún de su unilateralidad, la concebirá solo de una manera abstracta y terminará identificándola con la misma subjetividad del individuo (el bien y la consciencia moral en la "Moralidad”). En segundo lugar, aparecerá en escena la manera propia en que el bien (universal) será comprendido: el tercer momento del desarrollo del concepto de voluntad libre, es decir, la libertad realizada que tiene el título de "Eticidad".

Centrémonos, pues, en el primer punto. La voluntad subjetiva e individual ahora tiene una relación esencial con el bien universal, y se distingue, así, de las figuras que la precedieron en el capítulo de la "Moralidad" con respecto a los dos elementos que en ella se encuentran en relación: la subjetividad y la objetividad. Tanto el derecho de la subjetividad como el derecho de la objetividad adquieren aquí nuevas determinaciones. Por un lado, el derecho de la subjetividad ahora comprende "que lo que deba reconocer como válido sea considerado por ella como bueno" 3 , en ese sentido, de que no reconozca lo que no considera universalmente "racional". Este "derecho de apreciación de

2 Usamos, en castellano, la siguiente edición de la Filosofía del derecho: Hegel, G.W.F., Principios de la filosofía del derecho, traducción de Juan Luis Vermal, Barcelona: Edhasa, 1995 (Grundlinien der Philosophie des Rechts oder Naturrecht und Staatwissenschaft im Grundrisse, Werke in zwanzig Bänden, Frankfurt: Suhrkamp, 1970, tomo 7), § I29. En adelante citado como FD.

$3 F D, \S|3|$. 
lo bueno" se distingue de la voluntad moral que solo reconocía como propio de la acción lo que ella había presupuesto previamente en su propósito interior acerca de aquello que ha efectuado en el mundo ${ }^{4}$ (es decir, el derecho de saber). Pero también se distingue del derecho de la subjetividad a su bienestar, en tanto la referencia al bien que comporta esta última figura lo indica como siendo universal absolutamente, no teniendo, así, una forma relativa. No se trata ya solamente de la conservación en la acción del sujeto de un "propósito individual", ni del "bienestar entendido solo en términos subjetivos" 5 .

Por otro lado, el derecho de la objetividad ahora comporta una referencia a la acción que el individuo realiza en el mundo distinta a las anteriores: la acción de la subjetividad individual en el mundo, como una alteración del estado de cosas, exige su reconocimiento en este; para tenerlo, debe adecuarse a aquello que en el mundo se considera como válido ${ }^{6}$. Aquí, el mundo que reconoce la acción del sujeto como propia de este no es ya un mero mundo de condiciones naturales exteriores, en conciliación contingente con el propósito epistemológico del sujeto, ni un mundo de leyes abstractas y formales en contraposición eventual al bienestar vital del individuo, sino un mundo conformado por otras subjetividades, por otros sujetos racionales. Esta "subjetividad exterior", en la cual se conserva la subjetividad individual cuando realiza su fin a través de su acción y supera su recóndita e interior individualidad subjetiva, tiene que identificarse, así, con “la voluntad de los demás”. La realización del fin (la acción) del individuo comprende, entonces, la identidad de la voluntad individual y de la voluntad de los otros. Esto equivale a decir, finalmente, que "el terreno de la existencia de la voluntad es ahora la subjetividad". Hegel denomina a este dinamismo "subjetividad universal".

Las dos precisiones señaladas, así, comportan dos tesis claras. En primer lugar, en el terreno de la moralidad en su tercer corte ya se encuentra la referencia

$4 \quad F D, \S I I 7$.

5 Giusti, Miguel, "La lógica de la acción y los límites de la voluntad. Reflexiones sobre el concepto de 'acción' en Hegel”, en: Areté, III, No. 2 (199I), p. 225. El derecho del saber se refiere a la sección de "El propósito y la responsabilidad"; el derecho del bienestar, a la sección de "La intención y el bienestar".

$6 \quad C f . F D, \S 132$.

$7 \quad F D, \S I I 2$.

148 Cf. FD, § II2, obs. 
al bien universal en la misma subjetividad individual. En segundo lugar, el mundo se ha transformado ya, en una realidad social intersubjetiva conformada por otras subjetividades ${ }^{9}$. Pese a esto, la dinámica del desarrollo de las figuras comprendidas en esta sección de la "Moralidad" no permitirá tematizar, sino solo de manera negativa, el polo objetivo y exterior aquí señalado.

Como indicamos, es recién en la tercera sección de la "Moralidad" cuando ingresamos, después de los sentidos de acción moral revelados en los dos apartados precedentes, a aquella "voluntad subjetiva particular" que "quiere lo universal como tal”|", peculiaridad que se había anticipado al final de la sección titulada "Derecho abstracto", sin embargo, como la distintiva del punto de vista de la moralidad.

En primer lugar, Hegel presenta lo incondicionado del deber, identificado aquí con la filosofía práctica de Kant, y establece una crítica sobre este que conduce a la figura de la buena conciencia. El deber puro (o la ley moral), para Hegel, no solo no puede por símismo determinar deberes particulares, sino que, además, brinda la posibilidad de justificar cualquier proceder, sea moral o inmoral" . El deber puro, desvinculado de todo deber concreto, tiene como principio de discernimiento entre las máximas el establecer la falta de contradicción y, así, conduce a una "mera identidad formal”'2. El problema aquí, piensa Hegel, es que la contradicción puede implementarse como mecanismo de determinación de deberes solo si se asumen contenidos que cotejar. En otras palabras, la contradicción solo puede darse allí donde se parte de "un contenido que

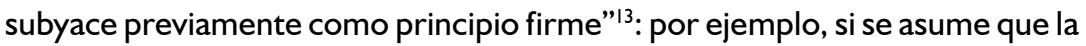
vida humana debe ser respetada a toda costa, se tiene un contenido en virtud del cual comparar y evaluar máximas de acción de acuerdo a si corresponden con o contradicen tal principio. Por ello, en la medida en que para el deber

9 Aunque no aparezca aquí su relevancia estructural, esta precisión es realmente importante y ha sido, según lo hemos visto, poco explotada. Michael Quante, por ejemplo, construye una teoría de la acción hegeliana limitándose solo al contenido de los momentos de la "Moralidad" previos a la implementación de esta referencia propiamente kantiana y moral (cf. Quante, Michael, El concepto de acción en Hegel, traducción de Daniel Barreto González, Barcelona: Anthropos, 2010).

$10 \quad F D, \S 130$.

II Cf. FD, § 135 , obs.

12 El imperativo categórico evidencia esto.

I3 FD, § I35, obs. 
puro, de acuerdo a Hegel, la herramienta de la falta de contradicción realmente no se puede implementar, pues se trata en la ley moral de una mera forma abstracta y universal, el vínculo entre la máxima (con contenido) y la ley (vacía), antes que permitir un discernimiento objetivo para la acción, se muestra como tautológico' ${ }^{14}$ : en última instancia, la forma universal, por su grado de abstracción, puede aguantar, antes que enjuiciar, el contenido del deber o de la máxima, sea el que sea. De esta manera, el sentido de la ley moral, como instancia enjuiciadora de deberes o máximas, parece invertirse en una instancia de autocomplacencia de los deseos e inclinaciones del sujeto.

Lo dicho da pie, inmediatamente, al tránsito hacia la buena conciencia. De acuerdo a lo señalado, se muestra que el criterio determinante para escoger entre una y otra máxima de acción asume necesariamente la referencia a su contenido $^{15}$. La ley moral carece de este y, por ello, no sirve como principio de determinación de contenidos: de ella no se derivan deberes concretos ${ }^{16}$. El hecho, sin embargo, es que de todos modos se actúa, y la actuación implica necesariamente deberes con contenidos concretos que se realizan en el mundo. Ya que la ley moral no permite, finalmente, escoger entre estos en virtud de su forma tautológica, la decisión, que tiene que darse, recae, finalmente, en la misma subjetividad de la conciencia, es decir, en "la absoluta certeza de sí misma"|", que es, así, la instancia que decide y determina qué se va a hacer en el mundo.

En términos históricos, mientras la figura del puro deber hace referencia al planteamiento de Kant sobre el imperativo categórico, la buena conciencia obtiene múltiples interpretaciones. En general, se trata del movimiento que extrema el principio moderno de la subjetividad, llevado antes a su máxima expresión por Kant, tarea que realizaron, desde distintos enfoques, Jacobi y Fichte, y que llega a comprender también la figura de la comunidad romántica ${ }^{18}$.

14 Cf. Giusti, Miguel, op. cit., p. 226.

I5 Cf. Siep, Ludwig, "¿Qué significa: «Superación de la moralidad en eticidad» en la «Filosofía del derecho» de Hegel?”, en: Amengual, Gabriel, (ed.), Estudios sobre la Filosofía «Filosofía del derecho» de Hegel, Madrid: Centro de Estudios Constitucionales, 1989, p. 180.

16 Cf. ibid.

$17 F D, \S 136$.

I8 Cf. Pinkard, Terry, Hegel's Phenomenology. The Sociality of Reason, Cambridge: Cambridge University, 1994, pp. 207 ss; Hyppolite, Jean, Génesis y estructura de «Fenomenología del espíritu de Hegel», traducción de Francisco Fernández, Barcelona: Ediciones Península, 199I, pp. 465-468. 
Conceptualmente, la distinción entre los dos conceptos puede darse a partir de la formulación del imperativo categórico kantiano y de su reformulación en Fichte. Mientras el primero señala que se debe actuar de acuerdo a una máxima que a la vez sea una ley universal, el segundo exige que se actúe de acuerdo a "la mejor convicción del deber; o bien, (...) según tu conciencia"|9. El primero defiende que algo debe ser máxima de la voluntad porque es principio de una legislación universal; invirtiendo los énfasis, el segundo señala que es justamente "porque algo debe ser máxima de mi voluntad" que "puede ser también principio de una legislación universal”20. El tránsito entre ambas figuras en la moralidad, así, puede leerse, como indica Giusti, como el desplazamiento del "peso de la contraposición" propia de la moralidad desde el polo objetivo del deber puro "hacia el polo subjetivo de la convicción subjetiva" ${ }^{21}$. Mientras que la referencia al deber puro es abordada a partir de una universalidad abstracta que, al no comportar posibilidad alguna de derivar deberes concretos, finalmente se trastoca en una forma autocomplaciente que puede recibir cualquier contenido ${ }^{22}$, la buena conciencia, en este marco, al ofrecer una profundización radical en el punto de vista de la moralidad, se presenta, por debajo de la universalidad del deber, como la instancia que decide a partir de su más profundad arbitrariedad subjetiva qué deberes concretos se deben realizar.

Antes de abordar la dinámica en la Fenomenología, debemos percatarnos de un último punto: el surgimiento de la buena conciencia comporta una ambigüedad que Hegel distingue. Habrá dos tipos de buena conciencia: una "verdadera" y "auténtica" 23 buena concienciay una buena conciencia solo formal. La distinción entre ambas recae para Hegel no estrictamente en la forma de cada una, pues ambas serán siempre una actividad de la voluntad subjetiva a partir de la cual

19 Cita de Fichte, en: Siep, Ludwig, op. cit., p. 180.

20 Cita de Fichte, en: Giusti, Miguel, op. cit., p. 227. Es interesante que la buena conciencia, aquí articulada con el planteamiento de Fichte, no es tematizada, finalmente, desde su referencia a la mediación intersubjetiva en instituciones sociales, como hace Fichte, sino solo rescatando la dimensión formal de tal conciencia, a saber, "el principio que establece la reflexión y la decisión del sujeto [la actividad de particularización]" (Siep, Ludwig, op. cit., pp. 180-18I), sin tomar cuenta lo concerniente a los contenidos morales de la acción.

21 Giusti, Miguel, op. cit., p. 226.

22 La crítica, así, se dirige ahí a la formalidad no motivante ni determinante del deber puro. Se puede, eventualmente, seguir aquí la categorización de Christopher Zurn.

$23 F D, \S 137$. 
esta decide qué hacer desde su propio fuero, sino en los contenidos por los cuales ella se decanta. La buena conciencia formal es la que él aborda como contraparte de la conciencia del deber abstracto. En esta buena conciencia formal, lo distintivo es solo la actividad de la subjetividad de acuerdo a la cual elige qué deber concreto realizar, sin tomar en cuenta el sentido propio del contenido de los deberes: por ello, se trata, en el fondo, de una arbitrariedad subjetiva que puede hacer tanto el bien como el mal. En este punto de vista, de esta manera, "la consciencia moral <buena conciencia> no tiene este contenido objetivo y es por sí la infinita certeza formal de sí misma, que por ello es al mismo tiempo certeza de este sujeto" ${ }^{24}$. Hegel la considera desde su unilateralidad sin tomar en cuenta su interdependencia con los contenidos que realiza: "en el punto de vista moral tal como ha sido diferenciado de la eticidad en este tratado, sólo entra la conciencia moral <buena conciencia> formal" 25 . Frente a esta, se encuentra la verdadera buena conciencia, la cual no se reduce al privilegio de la mera certeza de un deber, sino que es la convicción o la certeza de lo que es "en y por sí bueno"26. Una conciencia tal, sin embargo, solo surgirá en la eticidad y tenemos que ver los movimientos conceptuales que dirigen a este tercer estadio para llegar a comprenderla.

\section{§ 3. La buena conciencia como superación de la visión moral del mundo en la Fenomenología del espíritu: el momento previo a la eticidad}

En el caso de la Fenomenología, el tratamiento de la dinámica nos permite hacer más precisiones. El desarrollo del deber puro profundiza, a diferencia de lo que acontece en la Filosofía del derecho, en las tesis contradictorias que la conciencia que se autodetermina de acuerdo a la ley moral debe asumir con respecto a la naturaleza y los desplazamientos que son producto de su naturaleza contradictoria. En este marco, de manera, en principio opuesta, a como, en el caso de la Filosofía del derecho, la buena conciencia expresaba una profundización consecuente de la contraposición de la moralidad kantiana, aquí la buena conciencia es vista, más bien, como la solución a la contradicción

$\begin{array}{lll} & 24 & \text { lbid. } \\ & 25 & F D, \S \text { I37, obs. } \\ \text { I8 } & 26 & F D, \S \text { I37. }\end{array}$


inherente a la visión moral del mundo. La buena conciencia, de esta manera, es comprendida en la Fenomenología del espíritu como la superación de la antinómica visión moral. La tesis de Hegel acerca de tal superación (Aufhebung) por parte de la buena conciencia puede dividirse en dos afirmaciones. Por un lado, Hegel rescata la dimensión actuante y mundana de la buena conciencia. Por otro lado, la acción concreta de la buena conciencia tendrá que ser reconocida por las otras buenas conciencias en un orden social, un problema cuya solución a través del lenguaje no será idónea. En ese sentido, se requiere ver a la buena conciencia, en primer lugar, como una visión más completa de la visión moral del mundo para luego recién abordarla desde el aspecto formal que en la Filosofía del derecho se le suscribió. Pero, al elaborar este trámite, se evidenciará con mayor claridad aquello que se tiene que exigir para pasar al punto de vista de la eticidad.

La visión moral del mundo, que Hegel atribuye al planteamiento moral kantiano ${ }^{27}$, se fundamenta en dos tesis contradictorias que establecen una dinámica entre dos términos que se oponen $y$, sin embargo, mutuamente se exigen: el deber (la moralidad) y la naturaleza. La primera tesis afirma "la total indiferencia y la propia independencia" ${ }^{28}$ entre la naturaleza y la conciencia del deber. La autodeterminación del individuo en virtud de la ley moral, en efecto, exige una desvinculación por parte de este de cualquier exterioridad heterónoma. La segunda afirma, sin embargo, la absoluta esencialidad del deber y la "dependencia e inesencialidad de la naturaleza" ${ }^{29}$. La ley moral, pese a su independencia, debe llegar a ser la ley de la naturaleza, debe realizarse en el mundo y ejecutarse a través de la acción moral. Sobre esta base, la conciencia moral comportará intentos de resolver la contradicción entre ambas tesis estructurales $^{30}$ a través del planteamiento de postulados reconciliatorios.

27 Y que Siep considera que es complementado con el planteamiento fichteano (cf. Siep, Ludwig, El camino de la fenomenología del espíritu. Un comentario introductorio al Escrito sobre la diferencia y la Fenomenología del espíritu de Hegel, Barcelona: Anthropos, 2015, pp. 204-208; Siep, Ludwig, "Practical Reason and Spirit in Hegel's Phenomenology of Spirit", en: Moyar, Dean y Michael Quante (eds.), Hegel's Phenomenology of Spirit. A Critical Guide, Cambridge: Cambridge Universty Press, 2008, p. 18I).

28 Hegel, G.W.F., Fenomenología del espíritu, traducción de Wencesleao Roces, México D.F.: FCE, 20I0, p. 352. En adelante citado como FE.

29 Ibid.

30 Cf. Valls Plana, Ramón, Del yo al nosotros. Lectura de la fenomenología del espíritu de Hegel, Barcelona: PPU, 1994, p. 294. 
Estos, sin embargo, se mostrarán infructuosos y su afirmación comportará el desarrollo de los desplazamientos o deformaciones (Verstellungen) que configuran "el desarrollo de la autoconciencia moral en sus momentos" ${ }^{31}$. La complejidad conceptual de las contradicciones y desplazamientos, nos obliga, con miras a nuestro trabajo, a elaborar una breve síntesis de lo que en esta sección ocurre y extraer, asimismo, la doble oposición que Hegel halla en la visión moral del mundo.

El desplazamiento de la visión moral del mundo puede entenderse, sucintamente, de acuerdo a dos momentos que implican contradicción entre sus términos. En primer lugar, el deber puro exige cumplirse a través del actuar moral. Pero este cumplimiento implica propiamente el no actuar: uno actúa (moralmente) en tanto que no actúa (efectivamente), puesto que actuando limita la pureza de tal deber. Deber puro y acción, aunque se exigen mutuamente, son, sin embargo, mutuamente excluyentes. En segundo lugar, sin embargo, hay necesariamente que actuar. $Y$ en el momento de la acción, la conciencia descubre que hay otra cosa determinante para la acción: una realidad efectiva previamente dada y una realidad que se busca producir. Esto hace explícito la fundamental importancia de un contenido determinado (del fin y del deber concretos) como condición de posibilidad de toda acción. Pero así también se evidencia esto: cuando se realiza el deber moral o puro, no se le realiza, sino que se lleva a cabo un deber concreto. La conciliación entre ambos términos, en cada momento, es garantizada, finalmente, por una conciencia moral divina que se encuentra más allá de la conciencia moral existente.

Asimismo, hay, en la problemática de la visión moral del mundo, una doble oposición constitutiva que no debe pasar desapercibida. Se trata de la oposición, por un lado, entre moralidad y naturaleza, y, por otro lado, entre moralidad y sensibilidad, para cada una de las cuales la moralidad exige anclar un postulado que establezca una eventual identidad no presente entre ambos términos. El primer postulado (en sí) señala la "armonía de la moralidad y de la naturaleza objetiva" en "el fin último del mundo". El segundo postulado (para sí) señala "la armonía de la moralidad y de la voluntad sensible, el fin último de la autoconsciencia"32. La solución la ve Hegel, inmediatamente, en 
la mediación a través de la acción moral, que concilia en ambos casos los términos opuestos y establece una unidad existente. Pero, en la medida en que la moralidad es esencialmente contradictoria, una nueva contraposición al interior de ella conduce al surgimiento de un tercer postulado: un santo legislador del mundo que santifica, por un lado, los deberes determinados con relación al deber, y, por otro, el deber puro con relación a los contenidos de los deberes particulares ${ }^{33}$.

Frente a la antinómica visión moral, la buena conciencia (das Gewissen) es la conciencia moral actuante que sabe inmediatamente lo que es justo y debe realizarse. Comprende, de este modo, la conciliación del puro saber (lo universal) en la misma singularidad inmediata (lo particular) o el saber de lo real al mismo tiempo como "puro saber y puro deber" ${ }^{34}$. Hay que entender el posicionamiento de la buena conciencia en la cartografía de las figuras de la conciencia. Hegel, de hecho, le atribuye a esta tres distinciones que la permiten así precisarla. En primer lugar, a diferencia de la primera aparición del espíritu en el momento de la individualidad en y para sí misma (al final de la sección "Razón"), donde la cosa misma (die Sache selbst) era mero predicado, en la buena conciencia se ha asumido un proceso dialéctico que ha concluido ya en la identificación del espíritu con el sujeto que sabe los momentos anteriores como estando contenidos en él: la cosa misma (la substancia espiritual) se ha hecho sujeto plenamente. En segundo lugar, la buena conciencia se comprende como el "sí mismo" (das Selbst des Gewissens) que se distingue del sí mismo de la persona (al final de la sección sobre la eticidad en FE) y del sí mismo de la libertad absoluta (al final de la sección sobre la cultura). La persona es un sí mismo vacío que corresponde a una universalidad sin contenido. La libertad absoluta $^{35}$, al restituir la escisión del mundo de la cultura, corresponde a una realidad universal que, no obstante, "no tiene la forma de la existencia libre del sí mismo" ${ }^{36}$. La autoconciencia moral intenta darle la existencia libre, en tanto la vincula con la autodeterminación del sujeto particular de acuerdo a la forma del deber, pero mantiene una contradicción constitutiva entre sus tesis de fondo, en virtud de lo cual se tiene que experimentar un desplazamiento

\footnotetext{
33 Cf. Hyppolite, Jean, op. cit., p. 437.

34 FE, p. 369.

35 Propia del ciudadano revolucionario, como reconciliación de la universalidad y el sujeto (cf. Valls Plana, Ramón, op. cit., p. 306).
}

36 FE, p. 370. 
(Verstellung) que pasa de una tesis a otra de manera alterna sin establecer una plena identificación. Todas estas figuras, en fin, comprenden para Hegel un carácter relativo de vacuidad que es superado por la buena conciencia en tanto esta es la certeza de sí en un "contenido" que es "existencia misma" ${ }^{37}$

En tercer lugar, de manera específica, podemos abordar la buena conciencia en tanto inmediata superación de la visión moral del mundo y ver las implicancias de esta tesis. Tal superación puede verse como el tránsito de una conciencia moral inoperante o, en todo caso, contradictoria, a una conciencia moral resuelta y actuante. Como verdad de la autoconciencia moral, es superación (Aufhebung) de la oposición entre un fin puro como deber y una realidad entendida como "una naturaleza y una sensibilidad contrapuestas" 38 a tal deber: es "espíritu moral concreto" ${ }^{39}$ y su unidad está encarnada en "el momento mismo de la acción" 40 . Es, por ello, "actuación moral” que supera la moralidad inactiva. El punto de vista desde el cual se puede hacer explícita la superación de la oposición moral corresponde a la conciencia desplegada en su efectividad", al momento de la acción que, por ello, es "figura moral inmediatamente concreta" 42 : el puro deber y la naturaleza son "momentos superados", así, en esta "esencia moral que se realiza" ${ }^{* 3}$.

El concepto de la buena conciencia es leído, en esta primera presentación, desde la perspectiva de la realización de la acción. La ejecución de la acción supone que un determinado contexto de circunstancias (la gramática de la acción), un cierto curso de penetración en el mundo y un específico resorte sensible como impulso de tal penetración a través de la actuación han sido conjugados y desplegados por una subjetividad (un sujeto racional) que, decantándose por estos y no por otros, es decir, poniéndose a sí misma en ellos de manera determinada, los pone en práctica en la realidad efectiva. Por ello, la realización de la acción asume una actividad racional de autodeterminación por parte de un sujeto que se sitúa en un contenido específico en el cual

\footnotetext{
37 Ibid.

38 Ibid.

39 lbid.

40 Hyppolite, Jean, op. cit., p. 447.

41 Cf. ibid.

42 FE, p. 370.
}

2243 lbid. 
permanece, y que resulta en una resolución, es decir, una convicción de que algo (tal contenido) es aquello que debe ser realizado en el mundo.

Esta imbricación profunda entre el yo (lo universal) y la naturaleza (el contenido determinado de un querer y la gramática determinada de la situación en que tal querer impulsa a actuar) a través de una decisión actuante comprende dos consecuencias en el planteamiento de Hegel. En primer lugar, reporta claras ventajas explicativas sobre el terreno propio de la acción frente a la conciencia moral inactiva. En la buena conciencia, ahora, "el caso <el contexto y el contenido de la acción> es inmediatamente en la certeza sensible del saber como es en-sí y es en-sí solamente como es en este saber" ${ }^{\prime 4}$. Ya que no excluye mutuamente, sino que concilia en la unidad de la acción subjetiva tanto el saber y la certeza del deber con la particularidad de este, ella no consiste en un "medium positivo universal" 45 , donde cada una de las circunstancias de la acción adquiere el sentido de un deber substancialmente inconmovible, de modo que la acción no se realiza o es inmoral, ni en "una oscilante incertidumbre (Ungewißheit) de la conciencia”, que, haciéndose inmoral y actuante, pone la moralidad en una esencia sagrada o, conservando la moralidad para ella, pone en tal esencia la conexión de su moralidad con lo sensible.

Con todo, pese a comportar una superación de la visión moral del mundo, hay una segunda consecuencia no propiamente positiva. La buena conciencia contiene ya los recursos conceptuales para el desarrollo de una contraposición en su interior que se dirigirá a evidenciar las limitaciones de sus pretensiones. La buena conciencia es vista como una certeza subjetiva e íntima de algo que debe efectuarse porque se sabe que es un deber. En esta conciliación de forma y contenido están articulados ya "razón práctica" y "realidad concreta", pues en ella se asume, por un lado, la validez universal del deber $y$, al mismo tiempo, se tienen, por otro lado, las "indicaciones concretas para la acción" Ella es, claramente, decisión para actuar, en el fuero interno del sujeto, que se traduce inmediatamente en acción e introduce tal resolución en el mundo. En ese sentido, esta convicción íntima propia del sujeto, además, es pensada por

44 FE, p. 370. Por ello, la acción es la "forma pura de la voluntad" que tiene "realización" (FE, p. 370), algo que sirve también para exponer el carácter productor (creador) de la acción.

45 FE, p. 37I.

46 Siep, Ludwig, El camino de la fenomenología del espíritu, p. 209. 
este como la convicción que tendría cualquier sujeto racional (cualquier otra buena conciencia), de modo que si él la muestra a través de su acción, como de hecho hace, tendría, inmediatamente, la aprobación y la venia de los demás.

De acuerdo a lo dicho, se pueden ver las limitaciones de la buena conciencia. El tránsito de la visión moral del mundo a la buena conciencia no solo ha comportado una superación en clave de acción. La buena conciencia actuante comporta, también, una referencia universa ${ }^{47}$, de modo tal que la superación comprende además un tránsito en los sentidos de universalidad (del deber). La universalidad del deber de la visión moral del mundo, ciertamente, es abstracta e interior: solo en ella le es posible tematizar, propiamente, la referencia a la libertad humana. Consecuentemente, el mundo que se coloca frente a aquella es una naturaleza regida por leyes causales no animada por la libertad ${ }^{48}$. Pues bien, la dimensión universal propia de la convicción o certeza de la buena conciencia, donde queda conservado el deber de la sección anterior, no es ya el deber puro de la conciencia moral, sino que ahora es solo un momento de la unidad del sí mismo de la buena conciencia que, habiendo dejado de "ser esencia absoluta"49, dice Hegel, se identifica con el "ser para otro". Hegel establece la transición de un universal abstracto, interior y no existente, propio de la visión moral del mundo, a un universal concreto, objetivo y existente ${ }^{50}$, es decir, "inmediatamente real" 51 : "el momento del ser reconocido por los otros" 52 . La universalidad kantiana del deber interior se traslada a la naturaleza, $y$, en una suerte de amalgama, surge con claridad la realidad exterior que Hegel busca: el mundo social ${ }^{53}$. En efecto, el deber puro de la moralidad se ha traducido a la convicción subjetiva e íntima del deber,

47 Y un lado también particular. La certeza inmediata y concreta de sí es, al ser diferenciada, "tanto puro saber como saber de sí como de esta conciencia singular" (FE, p. 372).

48 Esto resulta bastante claro, por ejemplo, en el planteamiento de la tercera antinomia kantiana en la CRP, cuya resolución comprende pensar la libertad incondicionada solo en el plano de la idea. Valls Plana señala con precisión esto: "(...) el mundo al cual se opone la moral kantiana es un mundo natural, indiferente, carente de libertad” (Valls Plana, Ramón, op. cit., p. 30I).

49 FE, p. 373.

50 Cf. Hyppolite, Jean, op. cit., p. 460.

$5 \mathrm{FE}, \mathrm{p} .373$.

52 El ser para otro es deber. El deber es el "comportarse como universalidad respecto a otros", es "elemento común de las autoconciencias", es la "substancia en la que el acto tiene subsistencia y realidad efectiva" (FE, p. 373). Hyppolite, con razón, señala que aquí hay una "transición dialéctica bastante oscura" (Hyppolite, Jean, op. cit., p. 459). 
la cual, además, es asumida por el sujeto como la convicción universal (de las otras subjetividades) acerca del deber.

En este marco, la consideración de Hegel es que la buena conciencia da por sentado que su convicción y la convicción de las demás buenas conciencias acerca del deber se identifican plenamente, de modo que su acción en el mundo es pensada como reconocida inmediatamente por todos en tanto deber universal en el mundo social: "el saber de su fin es de modo inmediato el elemento de la existencia, el reconocimiento universal" 54 . Lo que se hace con convicción tendría ya que tener, de suyo, el reconocimiento de todos en la comunidad. Pero, no obstante, aparece una distinción entre ambos momentos $y$, entonces, la universalidad asumida como inmediata no es alcanzada: se da una inadecuación entre la convicción subjetiva del deber y la convicción de los demás ${ }^{55}$ que no permite que haya reconocimiento.

Una primera manera de entender la desigualdad es de tipo epistemológico. Siempre aparece alguna otra autoconciencia que, al asumir como relevantes otras circunstancias para el contexto de acción, pone en tela de juicio el saber de la buena conciencia sobre las circunstancias de la acción que esta realiza. De esta manera, el conocimiento que la buena conciencia había asumido como suficiente es revelado como relativo. Esto es así porque, finalmente, ninguna buena conciencia tiene un saber acerca del contexto o la situación que agote todas las circunstancias en que se actúa. El actuar, en tanto es realidad efectiva, comprende una pluralidad de circunstancias que se extienden de manera infinita en sus condiciones, en sus circunstancias concomitantes y en sus consecuencias, de modo que cualquier presunción de la buena conciencia acerca de un conocimiento total sobre el caso de la acción es inválida.

En segundo lugar, y esto es lo que consideramos ahora fundamental, se aprecia un desfase en la determinación de los contenidos considerados morales ${ }^{56}$. La buena conciencia asume que el deber en el cual ella tiene su convicción

54 FE, p. 373 y también cf. Hyppolite, Jean, op. cit., p. 460.

55 Cf. ibid., p. 461.

56 Se trata, por un lado, de un "defecto en el saber" de la buena conciencia y, por otro lado, de "un defecto sobre el contenido central de la acción" respectivamente (cf. Valls, Plana, Ramón, op. cit., p. 308). Este segundo punto es visto por Hyppolite desde el aspecto de "la determinación del deber" (Hyppolite, Jean, op. cit., p. 462). 
es un deber universal porque considera que las demás buenas conciencias comparten también esta misma determinación a obrar. En la medida en que se ha afirmado ya un mundo de otras autoconciencias, estas proceden también de acuerdo a tal principio. El desacuerdo surge cuando una buena conciencia considera un deber determinado como universal, pero otra, que procede de acuerdo al mismo principio, considera otro deber concreto como aquel que debe implementarse. Por ejemplo, la buena conciencia, que tiene como convicción el que acrecentar la propiedad es un deber puro, lo lleva a cabo de un cierto modo, a saber, a partir de los deberes determinados de "la conservación de sí mismo y de su familia" y "de ser útil a sus semejantes y de hacer el bien a los necesitados" 57 . Las otras autoconciencias, al considerar otros deberes particulares opuestos como los correctos, finalmente, diagnostican el modo de tal conciencia de realizar tal deber universal como incorrecto. De esta manera, "lo que aquéllos llaman cobardía es el deber de conservar la vida y la posibilidad de ser útil al semejante [para la buena consciencia]; y, por el contrario, lo que llaman valentía atenta contra estos dos deberes" ${ }^{58}$. En este marco, entonces, tampoco habrá una conciliación, pues la acción de la buena conciencia será criticada como inmoral o injusta. Pero, ipor qué surge tal desigualdad entre las buenas conciencias?

El contexto señalado asume un presupuesto que Hegel evidencia (a su manera): todas las buenas conciencias de este mundo social se consideran libres solo de acuerdo a la forma de su propia subjetividad y no de acuerdo a su sujeción a los deberes concretos (los contenidos para actuar) que eligen mediante su libertad subjetiva. En ese sentido, la universalidad que sostienen todas las buenas conciencias no corresponde propiamente a una universalidad constituida intersubjetivamente ${ }^{59}$.

Hegel explica esta tesis al señalar, ahora, que toda buena conciencia, cuando se encuentra en un contexto de acción, tiene que decidir entre una multiplicidad de deberes para actuar ${ }^{60}$. Esto quiere decir, por un lado, que la conciencia debe darle a la forma de su convicción alguno de los contenidos o deberes

\footnotetext{
57 FE, p. 376.

58 lbid.

59 Cf. Valls Plana, Ramón, op. cit., p. 309.

60 La buena conciencia es una decisión inmediata, casi un sentimiento, pero finalmente, una determinación de algo en vez de algo otro.
} 
que conforman tal multiplicidad; tiene que ponerse ella sobre un contenido particular, pues "ninguno es en su determinabilidad o en su contenido absoluto, sino solamente el puro deber"6।, entendido, de manera más precisa en la figura de la buena conciencia, como la "convicción del deber"62. Con esto se experimenta lo que en la Filosofía del derecho se había explicitado desde el inicio: la misma convicción del individuo, tomada ella de manera absoluta, "es tan vacía como el puro deber"63 propio de la visión moral del mundo. Pero, por otro lado, en tanto la buena conciencia es necesariamente actuante y la acción precisa siempre un contenido determinado, es necesario que este sea dado. Entonces, "la certeza inmediata de sí mismo" apunta a los "impulsos e inclinaciones", a la "sensibilidad"64, vistos finalmente como no conciliados sino como exteriores a la actividad racional de autodeterminación. Los contenidos del deber, al no poder ser deducidos ni del bien abstracto incondicionado ni de una realidad social intersubjetivamente libre, provienen, finalmente, de una naturaleza interior distinta a lo racional, a lo intersubjetivo, y carente de libertad. La buena conciencia, de esta manera, se evidencia como "una absoluta negatividad de todo lo determinado"65 que, sin embargo, en tanto es activa, "se determina por sí misma"66, es decir, siempre se da un contenido para obrar que, visto bien, tiene una forma racional solo exterior. Por ello, su verdad, finalmente, está supeditada a "la arbitrariedad del singular y la contingencia de su ser natural no consciente" 67 .

Ante este desfase, se intenta resolver los problemas del reconocimiento a través de un medio que aún no aparecía en esta figura: el lenguaje. Es a través del lenguaje como medio universal del reconocimiento que las buenas conciencias en este mundo social pueden expresar la convicción de su deber con inteligibilidad y mantener el sentido de su convicción o, en todo caso, especificar el sentido de la acción que han realizado. Si una acción es considerada como no deber, la buena conciencia puede emplear el discurso para hacer inteligible sus razones y alcanzar un acuerdo. Pero esta nueva

\begin{tabular}{ll}
\hline 61 & FE, p. 375. \\
62 & Ibid. \\
63 & Ibid. \\
64 & Ibid. \\
65 & Ibid. \\
66 & Ibid. \\
67 & Ibid., p. 376.
\end{tabular}


capa conceptual agregada a la acción comporta un gran nuevo peligro, en el que la buena conciencia cae: las buenas conciencias tienden, gracias al medio lingüístico, a considerar que lo único que vale en la comunidad es ya solamente la exposición de sus convicciones, y pierden de vista, de esta manera, el lado de la acción que corresponde, finalmente, al contenido, y, así, a la acción verdadera. Decir que se actúa no implica, necesariamente, el actuar en sentido estricto, y esta simple distinción en el sentido del actuar, pensamos, es la que Hegel quiere explotar. Dos consecuencias complementarias se develan, así, de este problema. En primer lugar, las buenas conciencias, refugiándose en el lenguaje, se hacen inactivas porque solo empiezan a hablar acerca de sus intenciones y dejan de obrar mediante la ejecución de tales intenciones. En esta dinámica, que Hegel concibe en un culto íntimo religioso, surge la figura del alma bella (die schöne Seele) ${ }^{68}$, una genialidad moral que no actúa para conservar la pureza de sus puras e interiores intenciones, ya que encuentra en ellas un vínculo directo con la divinidad, mientras que en la exterioridad se autocomplace exponiéndolas a través de un lenguaje que considera prolongación de su interioridad ${ }^{69}$, negando, además, la efectiva acción. En segundo lugar, en virtud del lenguaje, las buenas conciencias actuantes pueden ocultar las verdaderas intenciones de su acción, puesto que pueden brindar justificaciones que se articulan con el deber universal, cuando el resorte o motivo de su acción concreta, sin embargo, no lo hace. En ese sentido, el lenguaje, que aparece como capa conceptual de inteligibilidad en el mundo intersubjetivo de las buenas conciencias, sin embargo, conduce a una serie de problemas que no se logran resolver a partir del potencial explicativo que aquel tiene. La solución al problema del reconocimiento entre las buenas conciencias tendrá, finalmente, que ser otra.

68 FE, pp. 382-384.

69 Es a través de la capa conceptual del lenguaje que la buena conciencia actuante, finalmente, se puede convertir en un alma bella autocontemplativa. Esta es actuante por el lado del lenguaje, mas no en el sentido propio de realizar la intención que expresa en el lenguaje. 


\section{$\S$ 4. Tránsito de la moralidad a la eticidad: el sentido propio del contenido ético}

El análisis de la buena conciencia tanto en la Filosofía del derecho como en la Fenomenología del espíritu nos ha permitido extraer precisiones conceptuales importantes. En primer lugar, la buena conciencia es, por un lado, la profundización radical en el principio de la subjetividad, en la medida en que eleva la actividad de la subjetividad de decidir (el arbitrio) sobre lo bueno y lo malo a fundamento absoluto de todo deber. Pero, en segundo lugar, hemos visto, también, que la buena conciencia comporta una dimensión positiva que conduce a la conciliación de elementos contrapuestos a través de la realización de acciones efectivas en el mundo. En este, además, asume ya un mundo social en el que exige el reconocimiento de su acción como un deber universal por parte de las demás autoconciencias. En este marco, sin embargo, la buena conciencia adquiría nuevamente las características de una buena conciencia formal: haciéndose actuante, exigía un reconocimiento en el mundo que no se llegaba a dar puesto que su libertad se asumía solo a partir de su actividad subjetiva de autodeterminación para decidir arbitrariamente lo que era el deber. En ese sentido, la buena conciencia en ambos planteamientos comporta, desde dos tipos de acercamientos, la misma unilateralidad de un principio que, por ello, debe superarse. El norte de ella, como hemos visto, aparece encarnado en una "verdadera" y "auténtica" buena conciencia. La pregunta, entonces, es: ¿de qué manera la buena conciencia formal accede a esta nueva figura? $\mathrm{O}$ lo que es lo mismo: ¿de qué manera se da el tránsito de la (la libertad de la) moralidad a la (de la) eticidad?

En la Filosofía del derecho, por un lado, Hegel ha hecho explícito, como referencia a la universalidad, el concepto de bien, el cual, siendo la unidad entre la voluntad subjetiva y la voluntad universal, es comprendido desde el punto de vista moral todavía de manera abstracta, formal e indeterminada: es la "idea abstracta de bien" ${ }^{70}$. En última instancia, la referencia a tal bien abstracto, no articulado aún con el interés del individuo y con su particularidad, desemboca en la comprensión de aquel como el deber puro y en la exigencia de hacer el deber por el deber, identificándose con el planteamiento moral kantiano. Por 
otro lado, Hegel ha explicitado el lado particular de la moralidad articulado con tal universalidad abstracta. Naturalmente, el lado particular hacía referencia a los diferentes contenidos y deberes particulares a través de los cuales el sujeto operaba en el mundo. En ese sentido, tal particularidad, en última instancia, estaba referida al sujeto concreto que actuaba, puesto que este era el que, finalmente, disponía qué resortes sensibles, qué deseos, qué circunstancias para ingresar en el mundo, tenían que ser conjugadas y desplegadas en la acción. Pero, entonces, en la misma referencia a la particularidad, se develaba otra referencia universal, aunque, aislada en tanto tal, también abstracta: una "universalidad reflejada sobre sí"7l comprendida como una certeza absoluta e interior que se ponía arbitrariamente en un contenido o en otro, es decir, que determinaba y decidía qué hacer por sí misma desde su más íntima certeza de lo correcto. Esta distinción nos presentaba el concepto de buena conciencia (das Gewissen) formal.

Ambas figuras aparecen, al final del capítulo de la “Moralidad”, en un dinamismo dialéctico que Hegel despliega para establecer el paso hacia la siguiente figura de la voluntad: "Para el bien, por ser lo universal sustancial de la libertad de un modo aun abstracto, se requieren determinaciones y un principio de estas determinaciones que sean sin embargo, idénticos a él, del mismo modo que para la conciencia moral <buena conciencia>, el principio abstracto del determinar, se requiere la universalidad objetiva de sus determinaciones"72.

El bien, en tanto abstracto, no tiene determinaciones; la buena conciencia está siempre determinándose pero sin objetividad. Ambos, extremados en sus propios principios de manera unilateral, es decir, desde el punto de vista moral que gira en torno a la subjetividad, se presentan como abstracciones sin determinación que, sin embargo, deben ser determinadas. En primer lugar, puede darse ciertamente una identificación meramente unilateral ("en sí") entre ambos. Desde el punto de vista moral, el principio "de la subjetividad de la pura certeza de sí misma"73 pronto se descubre como idéntico finalmente a la "universalidad abstracta del bien"74. De esta manera, el deber

7I $F D, \S 136$.

$72 F D$, § |4I.

73 lbid. 
puro (o bien abstracto) y la actividad de autodeterminación del yo no son sino dos direcciones de una misma dinámica: se expresa lo mismo, finalmente, cuando se asevera que el deber puro, en vista de su formalidad y vacuidad, puede soportar cualquier contenido ${ }^{75} y$ cuando se afirma que el yo puro puede ponerse en cualquier contenido para obrar. $O$, como dice Siep, "son dos caras de la misma moneda"76: lo "incondicionalmente bueno" (el bien abstracto) se identifica con la "buena voluntad"77 (buena conciencia) a través de la pura indeterminación o la desvinculación de todo contenido. La pura subjetividad y el bien abstracto, así, concilian solo desde una dimensión parcial: la que no toma en cuenta, finalmente, ninguna determinación o, lo que es lo mismo, la que encuentra esta identidad en la profundidad de la interioridad del sujeto moral. La identificación en sí, o solo desde el punto de vista moral, de esta manera, tiene dos peculiaridades: es una identificación en una universalidad indeterminada, es decir, abstracta y es, también, una identificación en la pura interioridad del sujeto. No hay, pues, en tal unidad, ni contenido, ni exterioridad. Esto señala que la identidad formal del punto de vista moral pierde de vista, en esta última figura en la cual se consuma, el desarrollo de la acción, pues esta significa, precisamente, la exteriorización de un contenido particular ${ }^{78}$. Tal unidad indeterminada consiste, entonces, en una identificación que se da en la interioridad del sujeto moral $y$, en el interior de este, en la forma pura de su interioridad.

Pero, en segundo lugar, otra unidad ${ }^{79}$ (la conciliación en sí y para sí) puede darse si se adopta un punto de vista superior y más completo: a este nuevo punto de vista, como sabemos, Hegel lo denomina eticidad. La pregunta es: ¿de qué manera encuentra el bien sus determinaciones y la buena conciencia la objetividad (el bien) de sus determinaciones? Si nos percatamos de la presentación de la dinámica, ambos conceptos se exigen mutuamente: cada uno requiere, para realizarse, aquello que el otro tiene, pues tal atributo es

75 Y esto, en el fondo, es lo mismo que decir que rechaza cualquier contenido.

76 Siep, Ludwig, "¿Qué significa: «Superación de la moralidad en eticidad» en la «Filosofía del derecho» de Hegel?", p. I8I.

77 lbid., p. 182.

78 Para esto hay que recordar la definición de Hegel acerca de la acción: "la exteriorización de la voluntad como voluntad subjetiva o moral" (FD, §II3).

79 "Una identidad especulativa", señala Pippin en "Chapter six. The freedom of the will: social dimensions", en: Hegel's Practical Philosophy. Rational Agency as Ethical Life, Cambrigde: Cambrigde University Press, 2008, p. I5I. 
aquello de lo que carece y por cuya ausencia es aún indeterminado; entonces, de manera positiva, puede decirse que, si es que se reconoce la mutua carencia y la posibilidad recíproca en el otro de superar tal indigencia, puede realizarse un movimiento de intercambio a través del cual se genera la comunidad (la unidad) en la misma diferencia. El bien abstracto requiere de un principio de determinación para traducirse de manera efectiva en deberes concretos y en acciones ("debe ser"): para existir en el mundo necesita encontrarse en una conciencia actuante como su deber en concreto $y$, asimismo, realizarse propiamente a través de la acción de tal sujeto. La realidad del bien apunta, así, a la buena conciencia. La buena conciencia requiere para su ejercicio continuo de determinación, es decir, para actuar en el mundo a través de deberes concretos, sin embargo, un criterio universalmente objetivo que le permita superar la arbitrariedad de sus decisiones ("debe ser buena"). La buena conciencia tiene tal objetividad, naturalmente, en el bien universal. Tal identificación, si ocurre en el punto de vista moral, es parcial y, en ese sentido, no es propiamente una identificación, sino una reducción. Por ello, la mediación verdadera de tal identificación debe darse tomando en cuenta, necesariamente, aquello que en la primera identificación, como residuo, fue descartado: la misma determinación a través de la cual ya se realiza la acción en el mundo.

Con respecto a la Fenomenología $a^{80}$, en el último momento de la sección de la buena conciencia, Hegel presenta, en una última puesta en escena, una conciencia universal "para la que la esencia es más bien la universalidad, el deber $y$, en cambio, la singularidad (...) solo vale como momento superado" ${ }^{81} y$, por otro lado, una conciencia singular, para la que "la certeza de sí mismo es la esencia, frente al en sí o lo universal, que vale solamente como momento" ${ }^{82}$. Se trata, así, de la oposición entre una conciencia universal que enjuicia y no actúa y una conciencia singular que actúa, pero que no se adecua a lo universal en su acción. Por un lado, el sentido de la buena conciencia singular permanece con el sentido de la buena conciencia que hemos abordado como superación

80 Apelamos al capítulo del Espíritu de la Fenomenología para iluminar el tránsito de la moralidad a la libertad ética valiéndonos de la misma petición de Hegel (cf. FD, § I40). Siep también asume esta tesis (cf. Siep, Ludwig, “Practical Reason and Spirit in Hegel's Phenomenology of Spirit", pp. |7|-19|).

81 FE, p. 385.

282 Ibid. 
de la visión moral del mundo: una certeza inmediata acerca de lo que se debe realizar y que se traduce inmediatamente en acción en el mundo.

Por otro lado, la conciencia enjuiciadora comporta un variado registro de interpretaciones acerca de su sentido. En primer lugar, de acuerdo al tratamiento inmediatamente presentado, y en virtud de lo que el mismo Hegel enfatiza, la conciencia universal es una conciencia que se expresa lingüísticamente pero que, sin embargo, no actúa y, por ello, se identifica inmediatamente con el resultado al que nos condujo la implementación del lenguaje: el alma bella $a^{83}$. Sin embargo, pese a que la visión moral del mundo es muda y no tiene lenguaje, tal conciencia, después de todo, es una conciencia que busca preservar la pureza del deber universal sin empañarlo con ninguna acción a través de enjuiciamientos sobre el carácter determinado de esta. De acuerdo a esto, podemos señalar que la conciencia enjuiciadora universal es una manera de traducir, en una clave mundana e intersubjetiva que asume nuevos revestimientos conceptuales, la exigencia portada por el deber de la visión moral del mundo, el cual correspondía a una dimensión universal en el sujeto que no tenía cabida aún en el mundo, pues, finalmente, este era una naturaleza constituida por meras leyes causales. Siguiendo esa dirección, se puede señalar, con Siep, que en esta última sección del Espíritu, se hace presente el conflicto entre la decisión de la conciencia singular, que se considera soberana con respecto a las reglas morales y legales existentes, por un lado, y "la conciencia moral común articulada en leyes y juicios morales aceptados" ${ }^{84}$ y, en general, en "las costumbres vigentes" ${ }^{25}$, por otro lado. De esta manera, se trata, aquí, de la oposición y relación entre el individuo y el todo social ${ }^{86}$.

El desarrollo de Hegel ciertamente asume ya conceptos propiamente religiosos, avizorando el siguiente momento de la Fenomenología. Pero, con vistas a

83 Cf. FE, p. 390. Siep también asegura esto al abordarla como figura literaria en Jacobi, Novalis y Goethe (cf. Siep, Ludwig, El camino de la fenomenología del espíritu, p. 2I2).

84 Siep, Ludwig, Practical Reason and Spirit in Hegel's Phenomenology of Spirit, p. I8I. Hemos parafraseado la tesis de partida de Siep, quien anuncia acerca de esta última contienda: "La relación entre ambos lados es aún más complicada por una distinción y un conflicto dentro de la misma conciencia, a saber, entre la conciencia actuante y la conciencia enjuiciadora" (cf. ibid. Traducción nuestra). Las interpretaciones pueden ser múltiples y tal vez esa sea la intención de Hegel al final.

85 Siep, Ludwig, El camino de la fenomenología del espíritu, p. 212.

86 Cf. Siep, Ludwig, "Practical Reason and Spirit in Hegel's Phenomenology of Spirit", p. 183. 
nuestro trabajo, no vamos a dirigirnos por este camino. La dinámica dialéctica consiste en el reconocimiento de las propias limitaciones y unilateralidades (maldades) por parte de cada conciencia en las limitaciones (maldades) de la otra, en la medida en que se le brinda validez a tales limitaciones, lo que conduce a una renunciación por parte de cada una de su carácter sesgado. La buena conciencia singular y actuante decide de acuerdo a su propio punto de vista y, de esta manera, no se atiene, en absoluto, a los dictámenes del grupo social en el cual actúa, yendo, eventualmente, contra las costumbres y leyes vigentes: por esta razón, es considerada como mala ${ }^{87}$. La conciencia universal enjuiciadora y pasiva, por su parte, es mala en la medida en que siempre niega la validez de la realización de la acción por tener un carácter determinado y, en ese sentido, no se dirige ni se compromete con la aplicación de sus leyes o criterios $^{88}$. Cada una, prontamente, experimenta la unilateralidad de su propia perspectiva y pretensión a través del reconocimiento del valor de la pretensión y limitación de la otra. Cada una descubre, así, la necesidad de la limitación (y pretensión) de la otra y, por ello, renuncia a su limitación. En este marco, de esta manera, se debe reconocer que cada una adolece de una unilateralidad o indeterminación que solo supera en la medida en que acepta como válida la pretensión de la contraparte. La conciencia universal enjuiciadora "reconoce", así, que sus dictámenes solo pueden alcanzar concreción y, en ese sentido, se pueden aplicar en los contextos determinados de la realidad social a partir de la mediación de la decisión y acción propia de una buena conciencia. La conciencia singular actuante, asimismo, reconoce que sus acciones solo pueden ser inteligibles y entendibles en la realidad social si y solo si se atiene necesariamente, como Siep traduce, a "instituciones, valores y reglas comunes" ${ }^{89}$, a "comprensibles patrones de acciones" ${ }^{90}$. Con esto, se origina la relativización de ambas y surge la figura que Hegel denomina espíritu absoluto, la cual será abordada desde la figura de la religión. Solo en tal reconocimiento, señala Hegel, "las heridas del espíritu se curan sin dejar cicatriz"91.

De lo anterior, podemos rescatar dos conclusiones: solo a través de lo que hemos denominado la determinación en el obrar es que podrá darse la unidad

\footnotetext{
87 Cf. Siep, Ludwig, El camino de la fenomenología de espíritu, pp. 2II-2I2.

88 Cf. ibid., p. 212.

89 Siep, Ludwig, "Practical Reason and Spirit in Hegel's Phenomenology of Spirit”, p. 183.

90 Ibid.
}

34 9l FE, p. 39l. 
pertinente entre el principio del bien universal y el principio de autodeterminación de la buena conciencia; asimismo, la danza dialéctica del perdón entre las conciencias nos muestra que la conciencia universal enjuiciadora debe mediarse en la conciencia actuante para existir y, de manera recíproca, la buena conciencia singular debe limitarse a través de las exigencias de una conciencia universal que expresa el todo social para ella misma realizarse como tal. Pues bien, es el contenido propio de la eticidad el que comporta, en una respuesta final acerca de la libertad humana para Hegel, la determinación en el obrar como una dialéctica a través de la cual se median los dos principios en juego.

El bien viviente en que consiste la idea de la libertad, es decir, el contenido ético de la eticidad ${ }^{92}$, tiene que entenderse como una unidad concreta y negativa que consiste en la interdependencia dialéctica de dos dimensiones constitutivas, las cuales se realizan a sí mismas solo en la medida en que se median mutuamente, es decir, que alcanzan su plena realización solo en la medida en que se limitan recíprocamente. Tales dimensiones, al inicio del capítulo de la "Eticidad", son la autoconciencia y su acción individual ${ }^{93}$, las cuales conservan el derecho de la subjetividad a su libertad de autodeterminación -el lado propiamente particular en la estructura de la voluntad-, y las "instituciones y leyes" que constituyen una comunidad ética -lo que es la dimensión universal del concepto de voluntad-. Nuestra tesis explicativa sobre la eticidad, dicho esto, es la siguiente: el contenido ético, que es para Hegel la realización plena de la libertad universal o el sentido propio en que se expresa una voluntad plenamente libre, se entiende, desde un punto de vista objetivo, a partir de instituciones sociales y sus leyes inmanentes, todas las cuales expresan el bien universal, y las cuales existen como determinaciones subjetivas (intenciones individuales) para obrar de los miembros de la comunidad ética, mientras que, desde un punto de vista subjetivo, se comprende apelando a la existencia de individuos racionales modernos que se autodeterminan para actuar a partir de las determinaciones objetivas (leyes éticas) comportadas en las prácticas institucionales que conforman el todo social.

Enfatizando la relación de bicondicionalidad que se profesan ambas dimensiones, se puede decir que el bien universal, recolectado ahora en "las instituciones y

93 Esta no es otra que la buena conciencia. 
leyes existentes en y por sí" 94 , solo alcanza su realización, y se hace efectivo en el mundo en sentido estricto, si y solo si tales determinaciones son reconocidas por las autoconciencias que portan los miembros de la comunidad ética y en tanto estos, consecuentemente, las reproducen constantemente mediante sus acciones. Por su parte, los individuos o particulares que conforman una comunidad ética, a saber, las denominadas autoconciencias (donde se conserva, rearticulada en un nivel más pleno, el principio de la buena conciencia actuante), solo se realizan como tales en la medida en que, en efecto, asumen y llevan a cabo como sus propias (auto)determinaciones subjetivas tales leyes éticas. De esa manera, el individuo racional, solo al tener como determinaciones para obrar las determinaciones racionales propias de lo universal -las determinaciones éticas-, es que puede identificarse plenamente con estas; y solo al expresar lo universal a través de su acción -es decir, al desplegar aquellas determinaciones- es que se expresa realmente como voluntad individual o subjetiva.

El "contenido fijo" de lo ético solo es tal, de esta manera, precisamente porque corresponde a determinaciones objetivas para la acción que los miembros de la comunidad ética comportan como objetivos e intenciones propias que desplegar en el mundo. Si bien, en el capítulo de la "Eticidad", Hegel enfatiza en el aspecto objetivo del contenido ético, en tanto este, apreciado como un sistema de determinaciones racionales, coincide con los "poderes éticos" 95 comporta, así, la misma división del capítulo de la "Eticidad" 96 , no obstante, no debemos perder de vista que, por la misma constitución de la libertad ética, el elemento de la particularidad subjetiva ${ }^{97}$, de la autodeterminación y decisión de los individuos modernos, jamás es dejado de lado y, por el contrario, es necesariamente considerado como la condición de tal realización objetiva.

Es, precisamente, esta perspectiva subjetiva en la que ahora vamos a centrarnos para formular un último punto acerca del contenido ético. Desde esta

$94 F D, \S \mid 44$. Desde esta perspectiva, se trata de "la interconexión sustancial" propia del "sistema de determinaciones de la libertad" (Hegel, G.W.F, Enciclopedia de las ciencias filosóficas, traducción de Ramón Valls Plana, Madrid: Alianza Editorial, § 484).

$95 F D, \S \mid 45$.

96 En tanto implican la referencia a los momentos que acontecerán en el desarrollo de su concepto: la sucesión de los momentos (instituciones o esferas de acción) "Familia”, "Sociedad civil" y, finalmente, "Estado".

97 Es decir, los derechos del sujeto individual que son rescatados en la sección de la Moralidad son conservados en la libertad ética. 
perspectiva, que bien podríamos denominar inmanente o del participante, las "leyes éticas" aparecen con tres peculiaridades: reflejan las inclinaciones de los agentes, comportan una complementariedad para su ejecución y son principios intersubjetivos para la evaluación recíproca de acciones. La primera, ya sugerida, es un recurso indispensable que Hegel sostiene en su planteamiento para justificar por qué solo realizando la universalidad es que la particularidad se realiza a sí misma. Las últimas dos nos exigen ir más allá del planteamiento hegeliano en estos textos, pues nos conducen a señalar que las normas éticas cristalizadas institucionalmente no solo comportan la mediación para que el individuo se identifique con el orden social en general, sino que propician, de manera concomitante pero incluso más fundamental, relaciones de reconocimiento concreto ${ }^{98}$ entre los mismos miembros del mundo social.

En primer lugar, que el particular se realice solo a través de la universalidad, es decir, que los individuos se realicen en su individualidad solo en la medida en que se determinen de acuerdo a las normas éticas implica, para Hegel, la experimentación de una dinámica de resonancia con respecto a tales exigencias por parte de los mismos sujetos que participan de las prácticas de una comunidad ética. La consideración de Hegel es que, en una comunidad verdaderamente ética, los contenidos de la eticidad o leyes éticas aparecen, frente a los individuos, como teniendo "una autoridad absoluta" 99 y, asimismo, como no siendo "algo extraño", sino, finalmente, como un "testimonio del espíritu" 100 . Esto quiere decir que las leyes éticas que determinan los campos de acción de los sujetos aparecen ante ellos, si es que realmente son éticas, como deberes que, al constreñir en ciertos sentidos la voluntad de los individuos $^{101}$, no obstante, se presentan, ante ellos, como medios no arbitrarios, pertinentes $y$ deseables mediante los cuales estos pueden expresar y realizar su particularidad y, en ese sentido, alcanzar su propia "liberación"102. Los deberes éticos aparecen, ante los individuos, antes que como límites exteriores

98 Nos valemos aquí de la indicación de Siep, quien señala que el concepto de reconocimiento comporta una dinámica recíproca entre el sujeto y orden social (nosotros) y, asimismo, entre los mismos individuos (cf. Siep, Ludwig, "Practical Reason and Spirit in Hegel's Phenomenology of Spirit”, p. 183).

$99 F D, \S 146$.

100 Ibid., § 147.

I0I Cf. ibid., § 148 .

102 lbid., § 149. 
de su voluntad, como extensiones de su interioridad. De esta manera, "en el deber <ético> el individuo se libera y alcanza la libertad substancial"103.

Tal interdependencia entre las obligaciones para actuar y las propias inclinaciones o intereses particulares de los individuos ${ }^{104}$, de este modo, se debe a que la norma de la eticidad, como sostiene Honneth, tiene que reflejar un valor que "expresa las inclinaciones e intenciones de cada uno de los agentes" 105. Si esto es así, la sujeción a tal norma y las consecuentes obligaciones normativas que de aquí se derivan, como ya hemos señalado, no se ven como un constreñimiento extraño a los participantes en una praxis ética, sino como un medio para que puedan expresarse ellos mismos en su individualidad, es decir, para auto-realizarse y ser libres. De esta manera, los individuos que se sujetan a tales normas no encuentran un desfase entre aquello que desean y aquello que saben que deben hacer. Es así que "por medio de lo ético el hombre tiene derechos en la medida en que tiene deberes y deberes en la medida en que tiene derechos" 106 .

En segundo lugar, las normas o deberes de la eticidad se presentan en todas sus modalidades en la figura de objetivos que se realizan de manera complementaria ${ }^{107}$. Inauguran, de este modo, un ámbito de acción en el cual aquellos que ingresan en la práctica así determinada se experimentan como estando en un estado en el cual reconocen recíprocamente que solo pueden realizar sus propios propósitos si y solo si la contraparte en la interacción realiza los suyos ${ }^{108}$, de modo que exigen tal ejecución. Como ha señalado Axel Honneth, tales normas asumen las figuras de objetivos o intenciones "generales" de acción que satisfacen la condición de realizarse en una co-ejecución complementaria ${ }^{109}$, donde varias voluntades individuales se median recíprocamente exigiéndose

103 Ibid. Desde esta perspectiva, se trata de la "interconexión fenoménica" propia del "serreconocido", es decir, del hecho de que estas determinaciones se hacen valer en la "consciencia" (Hegel, G.W.F, Enciclopedia de las ciencias filosóficas, § 484).

104 Cf. Honneth, Axel, "The Normativy of Ethical Life", en: Philosophy and Social Criticism, vol. 40 (2014), p. 820.

105 lbid. Traducción nuestra.

106 FD, § 155.

107 Sigo a aquí a Honneth en El derecho de la libertad, traducido por Graciela Calderón, Buenos Aires: Katz Editores, 20I4, pp. 7I-72.

108 Cf. ibid., p. 67.

109 Cf. ibid., p. 72. 
actuar para realizarse a sí mismas. Si entendemos, como hemos señalado, el mundo exterior a nuestra subjetividad, es decir, el mundo objetivo, como un mundo constituido por otras subjetividades, acontece que el mismo mundo es el que exige que la intención que el individuo ha fraguado en su fuero interno sea ejecutada puesto que solo así tal mundo alcanza la satisfacción de sus propias intenciones ${ }^{110}$. Entonces, la exigencia para actuar no es interna ni pura, sino determinada y objetiva.

En tercer lugar, sucede que una norma ética desencadena un estado en virtud del cual los participantes en la práctica determinada por aquella norma adquieren, necesariamente, vinculaciones u obligaciones normativas para actuar en la medida en que se autorizan, de manera explícita o de manera implícita, el derecho de evaluar mutuamente sus desenvolvimientos o performances de acuerdo, precisamente, a los estándares normativos que asumen en tal práctica institucional. En ese sentido, las normas intersubjetivas de la eticidad se distinguen como principios de evaluación recíproca de las propias performances o acciones que los integrantes de tal relación institucional desempeñan en el orden social"' . Esto implica, además, que los participantes en tal interacción ética puedan considerarse como creadores de tal norma o principio en la medida en que son ellos mismos los que ofrecen, siempre, a través de su acción o de su juicio, una determinada interpretación de tal norma ética, la cual, además, puede variar en su inteligibilidad para los otros en diversos grados ${ }^{112}$.

Vista como recurso para una teoría de la acción subjetiva, la conceptualización de las normas éticas permite dar cuenta de una consecuente solución a la doble contraposición que constituye la voluntad individual, la cual presenta una inadecuación tanto entre el individuo racional y el mundo social como entre

110 Cf. ibid., pp. 7I-73.

III Con respecto a tal rasgo, Honneth señala que una relación de reconocimiento hace referencia: "A un conjunto especial de acciones sociales que tienen la siguiente propiedad: aquellos que llevan a cabo tales acciones se saben a sí mismos como estando sujetos a una cierta obligación normativa, la cual surge del derecho de los otros a juzgar tales acciones en referencia a una norma subyacente" (Honneth, Axel, "The Normativy of Ethical Life”, p. 819. Traducción nuestra).

II2 Todas estas condiciones, por citar un ejemplo, son satisfechas por la vinculación del amor, principio que inaugura la primera esfera de acción ética en el capítulo de la "Eticidad". 
la razón y el instinto ${ }^{113}$. En ese sentido, ya que, en virtud de los contenidos éticos, aquello que se distingue como deberes expresa para los individuos, a su vez, sus propias inclinaciones, la eticidad contiene, para Hegel, la solución del problema que acarrea la visión moral del mundo cuando separa entre razón y sensibilidad. Por su parte, las últimas peculiaridades señaladas satisfacen la condición de que el desenvolvimiento en el mundo no conduzca a un desfase irreparable entre el mundo y la intención del actor, pues es el mismo mundo el que exige que el sujeto actúe de acuerdo a su intención y solo actuando, finalmente, es que se distingue si su interpretación de la norma calza o no con las exigencias del mundo.

Al concebir la libertad como la unidad dialéctica de los contenidos éticos vigentes institucionalmente en la comunidad ética, el acercamiento sincrónico y estructural que hemos implementado exige rápidamente la implementación de una perspectiva diacrónica, o, más propiamente, histórica. Aquí solo podemos presentar una pequeña indicación al respecto. Hegel señala, en la Introducción, que "el mismo contenido que aquí se presenta bajo la figura de los instintos aparecerá más adelante <en la eticidad> en la forma de deberes" 14 . Como señala Pippin, las prácticas humanas han ido alcanzando a través de su desarrollo histórico "ciertos tipos de reflexión" o "ciertas consideraciones" sobre la manera de satisfacer los instintos que corresponden, en cuanto racionales, tanto con los principios del derecho abstracto y de la moralidad como con las tres formas de vínculo ético que se encuentran a la base de aquellos ${ }^{115}$.

II3 De esa manera, la doble contraposición que hemos señalado puede ser graficada, siguiendo a Wood, como teniendo, por un lado, un significado psicológico (la relación entre inclinación y razón) y, por otro lado, un significado social (la relación entre el sujeto particular y el conjunto de instituciones de la sociedad en la que actúa). Él mismo, sobre la base de esta distinción, establece una solución de esta doble problemática desde el punto de vista de la eticidad, solución que debe evidenciar el presupuesto de que ambas oposiciones no son sino dos aspectos de una misma contraposición (cf. Wood, Allen W., "Hegel's Critique of Morality”, en: Hegel, G.W.F., Grundlinien des Rechts der Philosophie, editado por Ludwig Siep, Berlin: Akademie Verlag, 1997, pp. 155-159).

II4 FD, § 19. "Más adelante" implica el momento de la eticidad. Asimismo, ya en la sección "Eticidad", Hegel sostiene que "el mismo contenido que adopta la forma de deberes y luego de virtudes tiene la forma de instintos" (FD, § I50, obs).

II5 Cf. Pippin, Robert, “Hegel, Freedom, The Will. The Philosophy of Right: §§ I-33”, en: Hegel, G.W.F., Grundlinien der Philosophie des Rechts, editado por Ludwig Siep, pp. 50-52. 
$\mathrm{Si}$, entonces, retornamos a la problemática surgida sobre el problema del reconocimiento entre las buenas conciencias ${ }^{116}$, nos percatamos de que tenemos las herramientas conceptuales para señalar por qué, en efecto, Hegel afirma que la imposibilidad del reconocimiento entre las buenas conciencias se explica en la medida en que, asumiendo unilateralmente el énfasis de la libertad en el lado meramente subjetivo e interior de la acción, no se llega a pensar ni a reconocer que el mismo contenido del deber, a saber, el deseo y el instinto, ha sido ya procesado y diseñado (históricamente) hasta haber alcanzado la forma de la libertad que refleja la libertad interior del individuo. Precisamente, adoptar la perspectiva de la Eticidad es, desde el corte que hemos privilegiado, asumir consecuentemente la naturaleza animada racionalmente de aquellas determinaciones que, al ser expuestas, si bien comportan la superación de las contradicciones de una libertad comprendida parcialmente, generan, a su vez, nuevas cuestiones acerca de su estatus que pueden ser tematizadas y respondidas solo si se parte en la investigación ya desde este punto de vista más completo sobre la libertad humana.

II6 Cf. ibid., final del apartado 3. 